(C) The Author(s), 2021. Published by Cambridge University Press on behalf of The Nutrition Society. This is an Open Access article, distributed under the terms of the Creative Commons Attribution licence (http://creativecommons.org/licenses/by/4.0/), which permits unrestricted re-use, distribution, and reproduction in any medium, provided the original work is properly cited.

\title{
Associations of dietary PUFA with dyslipidaemia among the US adults: the findings from National Health and Nutrition Examination Survey (NHANES) 2009-2016
}

\author{
Jiawei Zhou ${ }^{1}$, Lixin $\mathrm{Cai}^{1}$, Senmiao $\mathrm{Ni}^{1}$, Zihang Zhong ${ }^{1}$, Min Yang ${ }^{1}, \mathrm{Hao} \mathrm{Yu}^{1}$, Yang Zhao ${ }^{1}$, Pengcheng Xun ${ }^{2 * *}$ \\ and Jianling $\mathrm{Bai}^{1 *}$ \\ ${ }^{1}$ Department of Biostatistics, School of Public Health, Nanjing Medical University, 101 Longmian Avenue, Nanjing, Jiangsu \\ 211166, People's Republic of China \\ ${ }^{2}$ Department of Epidemiology and Biostatistics, School of Public Health-Bloomington, Indiana University, Bloomington, IN, USA
}

(Submitted 24 February 2021 - Final revision received 15 May 2021 - Accepted 10 June 2021 - First published online 21 June 2021)

\section{Abstract}

Dyslipidaemia, a significant risk factor of CVD, is threatening human health worldwide. PUFA are crucial long-chain fatty acids for TAG synthesis and removal, potentially decreasing dyslipidaemia risk. We examined dyslipidaemia prevalence among 15244 adults aged $\geq 20$ years from National Health and Nutrition Examination Survey 2009-2016. Dyslipidaemia was defined as total cholesterol $\geq 240 \mathrm{mg} / \mathrm{dl}$, or HDL-cholesterol $<40 \mathrm{mg} / \mathrm{dl} / 50 \mathrm{mg} / \mathrm{dl}$ for males/females, respectively, or LDL-cholesterol $\geq 160 \mathrm{mg} / \mathrm{dl}$, or TAG $\geq 200 \mathrm{mg} / \mathrm{dl}$, or taking lipid-modifying medications. We measured the daily PUFA intake using a 24-h dietary recall. Demographics, social economics, and lifestyle factors were collected using questionnaires/interviews. Additionally, we measured Se and $\mathrm{Hg}$ levels in the whole blood. Logistic regression models were used to examine the association between PUFA and dyslipidaemia. The unweighted and weighted dyslipidaemia prevalences were $72 \cdot 4 \%$ and $71 \cdot 0 \%$, respectively. When grouped into tertiles, PUFA intake above $19.524 \mathrm{~g} / \mathrm{d}$ was associated with an independent $19 \%$ decrease in dyslipidaemia risk $(\mathrm{OR}=0.81(95 \% \mathrm{CI} 0.71,0.94))$ compared with the lowest tertile (PUFA intake $\leq 12.349 \mathrm{~g} / \mathrm{d})$. A threshold inverse association was further determined by the restricted cubic spline analysis. When PUFA intake was increased to its turning point, that is, $19 \mathrm{~g} / \mathrm{d}$, the lower nadir risk for dyslipidaemia was obtained ( $\mathrm{OR}=0.72(95 \% \mathrm{CI} 0.56,0.89)$ ). When the exposure was the sum of $\alpha$-linolenic acid and octadecatetraenoic acid, the inverse linear association remained. Dietary PUFA intake is a beneficial factor for dyslipidaemia among American adults, independent of many potential confounders, including $\mathrm{Hg}$ and Se.

Key words: Dyslipidaemia: PUFA: Threshold association: National Health and Nutrition Examination Survey

Dyslipidaemia is one of the manifestations of the metabolic syndrome characterised by a change in the blood lipid profiles ${ }^{(1)}$ The accumulation of LDL-cholesterol, total cholesterol (TC) and TAG and the decrease of HDL-cholesterol are important risk factors of atherosclerotic plaques, which originate in the medium and large arteries, principally leading to $\mathrm{CVD}$ and $\mathrm{CHD}^{(1-3)}$. The increased prevalence and destructive power of dyslipidaemia are threatening human health worldwide ${ }^{(4,5)}$. From the epidemiological survey data, there were more than 100 million adults aged 20 years or older having TC levels of $200 \mathrm{mg} / \mathrm{dl}$ $(5.17 \mathrm{mmol} / \mathrm{l})$ or greater, and almost 31 million have levels of $240 \mathrm{mg} / \mathrm{dl}(6.20 \mathrm{mmol} / \mathrm{l})$ or greater from 2009 to 2012 in the $\mathrm{USA}^{(2)}$. In 2013 , the age-standardised mortality attributable to all CVD and CHD was 223.9 and 102.6 per 100000 persons, respectively, in the $\mathrm{USA}^{(6)}$. Traditionally, recognised risk factors for CVD included age, sex, obesity, hypertension, smoking status, type 2 diabetes, familial predisposition, and high levels of LDL-cholesterol ${ }^{(4)}$. Lowering LDL-cholesterol level with statin therapy can reduce CVD mortality ${ }^{(7,8)}$. Additionally, dyslipidaemia is also an essential consideration for controlling CVD risk ${ }^{(2,4)}$.

PUFA are fatty acids that contain more than one double bond in their backbone, generally including $n-3$ PUFA and $n-6$ PUFA $^{(9)}$. DHA, EPA, docosapentaenoic acid (DPA), $\alpha$-linolenic acid (ALA) and octadecatetraenoic acid (ODTA) are crucial long-chain $n$-3 PUFA for glucose and lipid metabolism as well as metabolic inflammation ${ }^{(10)}$. The mechanism of $n-3$ PUFA action, especially for DHA and EPA, is mediated by surface or

Abbreviations: ALA, $\alpha$-linolenic acid; DPA, docosapentaenoic acid; MEC, Mobile Examination Center; NHANES, National Health and Nutrition Examination Survey; ODTA, octadecatetraenoic acid; TC, total cholesterol.

* Corresponding authors: Pengcheng Xun, email pxun88@gmail.com; Jianling Bai, jbai@njmu.edu.cn 
intracellular fatty acid receptors and sensors. Intake of PUFA is associated with increased expression of adiponectin. As an anti-inflammatory cytokine, adiponectin promotes the hepatic metabolic enhancement and reduces the atherosclerosis risk by increasing HDL-cholesterol and reducing TAG. ALA and ODTA, often referred to as plant $n-3$ PUFA, are precursors of DHA and EPA. The physiological and functional attributes of plant $n$-3 PUFA appear to derive from their conversion to EPA or DHA via desaturase ${ }^{(11,12)}$. Dietary intake and supplements are the primary sources of $n-3$ PUFA in our daily life. Supplements are usually the derivatives with high EPA and DHA concentrations from fish, such as salmon, mackerel, or other oily fishes ${ }^{(13)}$.

Dietary habit changes with more unsaturated fatty acids are proposed to benefit metabolic disorders ${ }^{(12)}$. The beneficial effects of $n$-3 PUFA intake have been established widely in recent years ${ }^{(11,14,15)}$. Increased oily fish consumption was associated with a lower risk of hypertriacylglycerolaemia in the general Korean population ${ }^{(1)}$. In another randomised controlled trial study $^{(16)}$, 6-month use of DHA and EPA $900 \mathrm{mg} / \mathrm{d}$ was associated with a reduction in LDL-cholesterol and TAG levels. Furthermore, they observed an increase in HDL-cholesterol level. In a recent meta-analysis of studies totalling 693 CHD, type 2 diabetes or non-alcoholic fatty liver diseases, patients with an average age from 50 to 70 years old declared that high-dose $n-3$ fatty acids (purity $>90 \%$ ) slowed the atherosclerosis progression significantly, which is a potential mechanism in reducing CVD risk ${ }^{(17)}$. An authoritative advisory from the American Heart Association claimed that consuming non-fried seafood 1-2 times per week promotes a positive effect on the cardiovascular system, resulting in a reduced risk of cardiac death, $\mathrm{CHD}$, and ischaemic stroke ${ }^{(15)}$. In addition, PUFA promote effects on glucose and metabolic inflammation and as a good source of protein and vitamin $\mathrm{D}^{(12,15)}$. However, direct evidence on the association between dietary PUFA and dyslipidaemia risk among a nationally representative large sample-sized adults across various age groups is sparse if not lacking. The linear or non-linear association between dietary PUFA intake and dyslipidaemia is warranted to research.

Of note, fish consumption is a major source of long-chain PUFA intake. In addition to having PUFA, fish, as a package, also contains other trace minerals, including both beneficial elements (e.g., Se within a reasonable range) and toxic metals (e.g., Hg). se is an antioxidant that elicits its beneficial effects and may modify the association between $n$-3 PUFA and dyslipidaemia ${ }^{(18,19)}$. However, another study claimed that comparing the highest with lowest quartiles, the prevalence ratios of the Se-Zn pattern is 1.36 (95\% CI 1.13, 1.63) for the metabolic syndrome with National Health and Nutrition Examination Survey (NHANES) 2011-2014 ${ }^{(20)}$. This result corroborated with a study, which found that high se level is associated with increased TC, LDLcholesterol, HDL-cholesterol and $\mathrm{TAG}^{(21)}$. $\mathrm{Hg}$, which has heavy metal toxicity and accumulates along the ocean's food chain ${ }^{(19)}$, may enter the body along with PUFA. Some scholars suggested that $\mathrm{Hg}$ be evaluated in any participant with CHD or other vascular diseases ${ }^{(22)}$. Another research indicated that $\mathrm{Hg}$ influences include thrombosis, immune and mitochondrial dysfunction, and dyslipidaemia ${ }^{(23)}$. Thus, it deserves an investigation whether
Se and $\mathrm{Hg}$ will confound and modify the association between dietary PUFA and dyslipidaemia risk.

Therefore, in this study, we aim to examine the associations between the consumption of PUFA and the risk of dyslipidaemia, and whether the associations will be confounded and modified by se and $\mathrm{Hg}$ levels in the US population based on NHANES 2009-2016 data.

\section{Materials and methods}

\section{Study population}

The NHANES is a nationally representative measurement of the civilian non-institutionalised US population with a stratified multistage probability cluster design conducted by the National Center for Health Statistics of the Centers for Disease Control and Prevention. For this study, four NHANES survey waves (2009-2010, 2011-2012, 2013-2014, and 2015-2016) were combined using adjusted sampling weights. A total of 40439 individuals were selected from these four waves. Eligible participants were 23234 adults aged 20 or older, with no missing information about sex, ethnicity, education, and weight. Seventeen participants were excluded due to missing smoking and diabetes information. We excluded 2624 missings on the PUFA who did not complete the two 24-h dietary recall interviews. These exclusions resulted in the final sample size of 15244 adults after 5349 participants were eliminated due to missing measurements on the laboratory examinations, including TC, HDL-cholesterol, LDL-cholesterol, and TAG, or dyslipidaemia medication. The detailed sample elimination process is shown in Fig. 1.

\section{Measurement of dyslipidaemia}

Blood dyslipidaemia was defined as $\mathrm{TC} \geq 240 \mathrm{mg} / \mathrm{dl}$, or HDL-cholesterol $<40 \mathrm{mg} / \mathrm{dl}$ for males, HDL-cholesterol $<50$ $\mathrm{mg} / \mathrm{dl}$ for females, or LDL-cholesterol $\geq 160 \mathrm{mg} / \mathrm{dl}$, or TAG $\geq 200 \mathrm{mg} / \mathrm{dl}$, or self-reported usage of prescribed lipid-modifying medication. The TC, HDL-cholesterol, LDLcholesterol and TAG information all derived from the laboratory measurements. The biospecimens were collected at the Mobile Examination Center (MEC), including the collecting, processing, storing and shipping of blood specimens. The MEC's controlled environment allowed laboratory measurements to be done under identical conditions at each survey location ${ }^{(24)}$.

\section{Measurement of PUFA}

In the NHANES study, the dietary intake information was collected by the trained interviewers for all NHANES examinees and used to estimate the types and amounts of foods and beverages (including all types of water) consumed during the 24$\mathrm{h}$ period prior to the interview (midnight to midnight) and to estimate intakes of energy, nutrients and other components from those foods and beverages. Daily intake of PUFA was reported from the average of two 24-h dietary recall interviews. The first dietary recall interview was collected in-person at the MEC, and the second interview was conducted by telephone after 3-10 d, but not on the same day of the week as the first MEC interview. If 


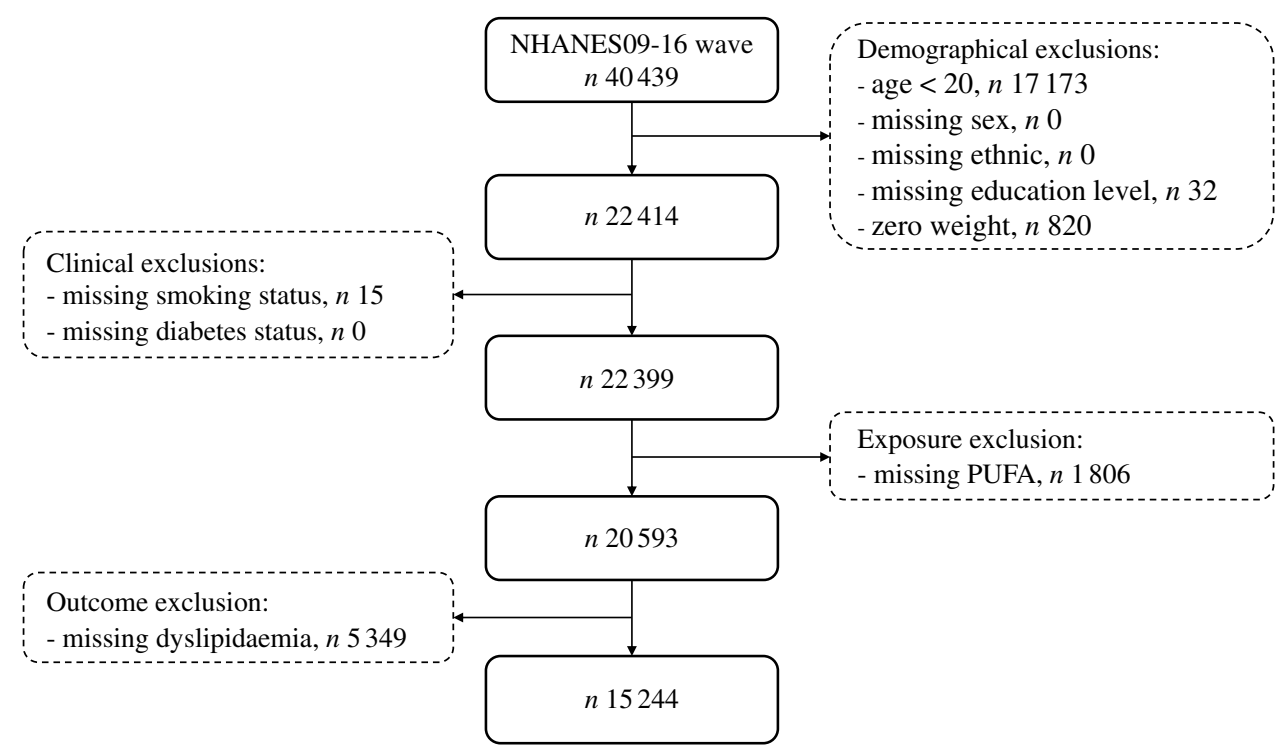

Fig. 1. Flow chart of data exclusion process of this study.

a participant did not finish the second dietary call interview, only the first dietary interview was used as average. Daily intakes of the common long-chain $n$ - 3 fatty acids, DHA, EPA, and DPA, and their sum were calculated for each participant from the average of two 24-h dietary recall interviews. Other two $n$-3 unsaturated fatty acids, ALA and ODTA, and their sum were also obtained from the interviews.

\section{Measurement of mercury and selenium}

The measured $\mathrm{Hg}$ and Se were derived from blood specimens. Whole blood specimens were collected at the MEC, processed, stored, and shipped to the Division of Laboratory Sciences. Some measurements below the detection limit were imputed with the value of 'detection limit $/ \sqrt{ } 2^{\text {(25) }}$. In the wave of the year 20092010, all specimens were not detected se in the serum. $\mathrm{Hg}$ was measured for all four waves. The $\mathrm{Hg}$ and se in $\mu \mathrm{g} / \mathrm{l}$ were converted to $\mathrm{nmol} / 1$ by multiplying 4.99 and $0.0127 \mathrm{nmol} / \mu \mathrm{g}$, respectively.

\section{Potential confounders}

In the NHANES study, demographic, behaviour, social-economic information, and clinical status could be obtained from questionnaires and examinations. In our research, we considered the following variables as potential confounders: age group (groups for every 15 years starting from 20 years old), ethnicity (non-Hispanic white, non-Hispanic black, Hispanic, and others), sex (male and female), BMI, education (under senior high, senior high, and college or university and above), smoking status (never, past, and current), alcohol beverages (total alcoholic drinks per year classified by median), diabetes status (non-diabetes and diabetes) and hypertension (yes $v$. no). We adjusted these confounders in multiple models sequentially, which would be detailed in the statistical analysis part.

\section{Statistical analysis}

Accounting for the complex, stratified, multistage cluster sampling design structure, we used survey procedures in SAS statistical software version 9.4 (SAS Institute Inc.) for all statistical analyses. Four waves of continuous survey data were combined, and an 8-year sampling weight was calculated for the analyses.

Descriptive statistics, including counts and percentages for categorical variables, weighted means and standard errors for continuous variables, were calculated for dyslipidaemia and regular lipid group, respectively. Due to the highly skewed distribution of se and $\mathrm{Hg}$, we computed weighted geometric means and $95 \%$ CI for these variables ${ }^{(26)}$. For all PUFA, the weighted median with inter-quartile range was calculated in two groups, based on their sampling distributions.

We constructed multivariable logistic regression models with adjusting confounders to investigate the OR as well as 95\% CI for the risk of dyslipidaemia in association with PUFA. In model 1 , we adjusted for age, sex, and race; model 2 further adjusted for education, BMI, smoking status, and alcoholic beverages; model 3 additionally adjusted for hypertension and diabetes. Also, the intakes of protein and cholesterol were involved in model 4 . In model 5, we mainly considered the effects of PUFA after adjusting the impact of blood $\mathrm{Hg}$ and se.

We also fitted PUFA with restricted cubic spline functions to explore the non-linear association with the original data. Both first- and second-order interactions among PUFA intake, blood se $(\geq v .<$ median $)$, and $\mathrm{Hg}(\geq v$. $<$ median) were considered in the full model. The stratification analyses were done for each $\mathrm{Hg}$ and se level to explore the effect modification.

Considering the potential impact of missing data on the analysis results, we performed multiple imputation, mainly for missing values of se and $\mathrm{Hg}$. We performed logistic regression models to consider the OR for different intake levels of PUFA and 
interactions with imputed data for the main analysis. All logistic regression results of imputed data were obtained using PROC MIANALYZE in SAS for considering the imputation effect.

\section{Results}

Study population characteristics are presented in Table 1 . The unweighted prevalence of dyslipidaemia was $72.4 \%$, with 11041 interviewees classified as dyslipidaemia among a total of 15244 participants. Participants with dyslipidaemia were more likely to be older, smokers, diabetic, non-Hispanic whites, and had higher BMI and blood pressure and lower education than those without dyslipidaemia. Lower intakes of PUFA, protein, DPA, ALA, ODTA, the sum of ALA and ODTA, and Hg were also observed in dyslipidaemia participants. As for sex, the consumption of alcoholic beverages, intakes of cholesterol, DHA, EPA, the sum of DHA, EPA, and DPA, and se, there was no difference between participants with or without dyslipidaemia.

An inverse association was found between PUFA intake and dyslipidaemia (Table 2). Comparing with those in the lowest tertile of PUFA intake, those in the middle and upper tertiles had a $17 \%(\mathrm{OR}=0.83(95 \% \mathrm{CI} 0.75,0.93))$ and $19 \%(\mathrm{OR}=0.81(95 \%$ CI $0 \cdot 71,0.94))$ lower risk, respectively, after controlling for multiple confounders ( $P$ for linear trend $=0 \cdot 008$ ). The inverse association was further confirmed when PUFA intake was fitted using restricted cubic spline functions (Fig. 2(a)). Compared with the lowest PUFA intake, a threshold inverse association of PUFA with dyslipidaemia was observed. A nadir risk for dyslipidaemia was reached $(\mathrm{OR}=0.72(95 \% \mathrm{CI} 0.59,0.86))$ when PUFA intake arrived at its tuning point of $19 \mathrm{~g} / \mathrm{d}$.

When the exposure of interest was the sum of intakes of ALA and ODTA, the inverse association remained (T3 $v$. T1: $\mathrm{OR}=0.87(95 \%$ CI $0.76,0.99) ; P$ for linear trend $=0.04)$ (Table 3). However, we did not observe a threshold phenomenon while fitting the exposure with restricted cubic spline functions (Fig. 2(b)).

We found that neither se $(\geq v$. $<$ median) nor $\mathrm{Hg}(\geq v$. $<$ median) modified the associations of interest in both the categorical analyses and the restricted cubic spline analyses (results not shown). There was no interaction between se, $\mathrm{Hg}$, and PUFA intake, either. Stratification analysis by sex was also performed for effect modification exploration. The associations were consistent between males and females.

\section{Discussion}

In this cross-sectional study of a nationally representative sample of the US adults using data from the NHANES 2009-2016, we found inverse associations of both intakes of PUFA, and the sum of ALA and ODTA with the risk of dyslipidaemia, after controlling for some potential cofounders including blood se and $\mathrm{Hg}$.

The inverse relation between PUFA and dyslipidaemia was consistent with the findings from previous studies ${ }^{(11,14-17)}$. The $n$-3 PUFA intake operated on the decrease of plasma TAG, VLDL, and APOB-100, as well as the increase of $\mathrm{HDL}^{(12,27-29)}$ The potential mechanisms of $n-3$ PUFA exerting effects are as follows. $N$-3 PUFA may decrease the expression of sterol regulatory element-binding protein- $1 \mathrm{c}$, contributing to reduced expression of cholesterol-, fatty acid-, and TAG-synthesising enzymes. They could also increase the mitochondrial oxidation rates, or peroxisome, resulting in a reduction in available substrate required for TAG and VLDL synthesis. In addition, they have been shown to inhibit key enzymes involved in hepatic TAG synthesis and increase the expression of lipoprotein lipase, leading to decreased TAG synthesis and increased TAG removal from circulating VLDL and chylomicron particles ${ }^{(12,27,28,30)}$. Some studies reported that $n-3$ PUFA reduced serum TAG in a population with hypertriacylglycerolaemia and increased LDLcholesterol and HDL-cholesterol; however, the increase in LDL-cholesterol was less than the reduction in VLDL-cholesterol resulting in a decrease of non-HDL-cholesterol (VLDLcholesterol and LDL-cholesterol) ${ }^{(31,32)}$.

However, the large-scale randomised controlled trial studies - VITAL (NCT01169259) ${ }^{(33)}$ and ASCEND $\left(\right.$ NCT00135226) ${ }^{(34)}$ - revealed that there was no significant difference in the incidence of major cardiovascular events between the $n$ - 3 fatty acid supplementation group and the placebo group. In these two studies, the main exposure included $n$ - 3 fatty acids 1 $\mathrm{g} / \mathrm{d}$. But in the VITAL research, a lower incidence of the primary cardiovascular endpoint in the $n-3$ supplementation group could be observed in the low fish consumption strata ${ }^{(33)}$. In fact, the resources of daily PUFA are derived not only from supplementation but also from dietary seafood. Thus, the biologically plausible effect of PUFA raised the question of the potential difference between cardiovascular events and intermediate cardiovascular endpoints, such as dyslipidaemia, diabetes, inflammatory reaction, and hypertension.

The other two large-scale randomised controlled trials REDUCE-IT (NCT01492361) ${ }^{(35)}$ and JELIS (NCT00231738) ${ }^{(36)}$ showed that highly purified EPA would reduce the incidence of cardiovascular events in patients who had been under a statin therapy for treating hypertriacylglycerolaemia or hypercholesterolaemia. Both trials claimed that EPA was efficacious for participants who had established CVD for secondary prevention. What is noteworthy was that the imbalanced sex ratio and high fish consumption diet failed to detect a significant effect on primary prevention with an underpowered analysis in a Japanese study ${ }^{(36)}$. Based on a high PUFA diet, purified EPA was also useful as a prescription medicine for secondary prevention. Pharmacological interventions and nutritional observations are difficult to inter-extrapolate because PUFA contain many fatty acids other than EPA. Further food-based or nutrient trials are warranted to explore the dietary PUFA effect on primary or secondary prevention of different types of CVD.

To our knowledge, this study was the first large-scale observational research on the associations between dietary PUFA and dyslipidaemia risk using the NHANES data. We clearly and newly illustrated the linear relation and a threshold phenomenon between PUFA intake and dyslipidaemia risk. The benefits of dietary PUFA on dyslipidaemia have a potential translative value to the treatment and prevention of hyperlipidaemia in clinical practice. This study further considered se and Hg's potential confounding effect due to PUFA intake often deriving from deep ocean oily fish. In participants with higher Hg levels in the blood, 
Table 1. Characteristics of study participants, by dyslipidaemia status, a cross-sectional study using data from the NHANES $2009-2016$ (numbers and percentages).

\begin{tabular}{|c|c|c|c|c|c|c|c|}
\hline \multirow[b]{2}{*}{ Characteristics } & \multicolumn{2}{|c|}{$\begin{array}{l}\text { All participants } \\
\text { (n 15 244) }\end{array}$} & \multicolumn{2}{|c|}{$\begin{array}{l}\text { Participants with } \\
\text { dyslipidaemia } \\
(n 11041)\end{array}$} & \multicolumn{2}{|c|}{$\begin{array}{l}\text { Participants without } \\
\text { dyslipidaemia } \\
\text { (n 4203) }\end{array}$} & \multirow[b]{2}{*}{$P^{\star}$} \\
\hline & $n$ & $\% \dagger$ & $n$ & $\% \dagger$ & $n$ & $\% †$ & \\
\hline Male & 7328 & $48 \cdot 20$ & 5300 & $48 \cdot 25$ & 2028 & 48.07 & 0.87 \\
\hline \multicolumn{7}{|l|}{ Race/ethnicity } & 0.002 \\
\hline Non-Hispanic white & 6471 & $67 \cdot 88$ & 4779 & $68 \cdot 70$ & 1692 & $65 \cdot 87$ & \\
\hline Hispanic & 4079 & $14 \cdot 62$ & 3036 & $14 \cdot 60$ & 1043 & 14.65 & \\
\hline Other & 1735 & $7 \cdot 44$ & 1165 & $7 \cdot 12$ & 570 & $8 \cdot 22$ & \\
\hline \multicolumn{7}{|l|}{ Education } & $<0.001$ \\
\hline Less than high school & 3830 & $16 \cdot 80$ & 2959 & $17 \cdot 84$ & 871 & $14 \cdot 26$ & \\
\hline High school graduate & 3396 & $21 \cdot 84$ & 2548 & $22 \cdot 71$ & 848 & $19 \cdot 74$ & \\
\hline Some college or above & 8018 & $61 \cdot 35$ & 5534 & 59.45 & 2484 & $66 \cdot 00$ & \\
\hline \multicolumn{7}{|l|}{ Smoking status } & $<0.001$ \\
\hline Non-smoker & 8375 & 54.91 & 5837 & 52.97 & 2538 & 59.66 & \\
\hline Hypertension & 8288 & $51 \cdot 33$ & 6743 & $58 \cdot 75$ & 1545 & 33.11 & $<0.001$ \\
\hline Diabetes & 3206 & $16 \cdot 50$ & 2803 & 20.43 & 403 & $6 \cdot 90$ & $<0.001$ \\
\hline \multicolumn{7}{|l|}{ Age (years) } & $<0.001$ \\
\hline Weighted mean & \multicolumn{2}{|c|}{$49 \cdot 26$} & \multicolumn{2}{|c|}{51.54} & \multicolumn{2}{|c|}{43.69} & \\
\hline Standard error & \multicolumn{2}{|c|}{0.29} & \multicolumn{2}{|c|}{0.28} & & & \\
\hline $\operatorname{BMI}\left(\mathrm{kg} / \mathrm{m}^{2}\right)$ & & & & & & & $<0.001$ \\
\hline Weighted mean & & & & & & & \\
\hline Standard error & & & & & & & \\
\hline Alcohol beverage consumpt & ic drinks & & & & & & 0.45 \\
\hline Weighted mean & & & & & & & \\
\hline Standard error & & & & & & & \\
\hline Systolic blood pressure $(\mathrm{mm}$ & & & & & & & $<0.001$ \\
\hline Weighted mean & & & & & & & \\
\hline Standard error & & & & & & & \\
\hline Diastolic blood pressure (m & & & & & & & $<0.001$ \\
\hline Weighted mean & & & & & & & \\
\hline Standard error & & & & & & & \\
\hline Standard error & & & & & & & \\
\hline Cholesterol intake (mg/d) & & & & & & & 0.49 \\
\hline Weighted mean & & & & & & & \\
\hline Standard error & & & & & & & \\
\hline PUFA (g/d) & & & & & & & $<0.001$ \\
\hline Weighted median & & & & & & & \\
\hline 25th and 75 th percentiles & & & & & & & \\
\hline $\mathrm{DHA}(\mathrm{mg} / \mathrm{d})$ & & & & & & & 0.12 \\
\hline Weighted median & & & & & & & \\
\hline 25th and 75 th percentiles & & & & & & & \\
\hline EPA (mg/d) & & & & & & & 0.22 \\
\hline Weighted median & & & & & & & \\
\hline 25th and 75 th percentiles & & & & & & & \\
\hline Docosapentaenoic acid (mg & & & & & & & 0.03 \\
\hline Weighted median & & & & & & & \\
\hline 25th and 75th percentiles & & & & & & & \\
\hline $\mathrm{DHA}+\mathrm{EPA}+$ docosapentae & $\mathrm{ng} / \mathrm{d})$ & & & & & & 0.11 \\
\hline Weighted median & & & & & & & \\
\hline 25th and 75 th percentiles & & & & & & & \\
\hline$\alpha$-linolenic acid (mg/d) & & & & & & & $<0.001$ \\
\hline Weighted median & & & & & & & \\
\hline 25th and 75 th percentiles & 101 & 3.32 & 993 & 3.35 & 107 & 2.77 & \\
\hline Octadecatetraenoic acid (m & & & & & & & 0.02 \\
\hline Weighted median & & & & & & & \\
\hline 25th and 75th percentiles & & & & & & & \\
\hline$\alpha$-linolenic acid + octadecat & sid (mg/ & & & & & & $<0.001$ \\
\hline Weighted median & & & & & & & \\
\hline 25th and 75 th percentiles & 102 & 8.00 & 1002 & $9 \cdot 73$ & 10 & $5 \cdot 27$ & \\
\hline
\end{tabular}


Table 1. (Continued)

\begin{tabular}{|c|c|c|c|c|}
\hline \multirow[b]{2}{*}{ Characteristics } & $\begin{array}{l}\text { All participants } \\
\text { (n 15 244) }\end{array}$ & $\begin{array}{l}\text { Participants with } \\
\text { dyslipidaemia } \\
(n 11041)\end{array}$ & $\begin{array}{l}\text { Participants without } \\
\text { dyslipidaemia } \\
\text { (n 4203) }\end{array}$ & \multirow[b]{2}{*}{$P^{\star}$} \\
\hline & $\% \dagger$ & $\% \dagger$ & $\% \dagger$ & \\
\hline Blood Se $(\mu \mathrm{g} / \mathrm{l}) \ddagger$ & & & & 0.88 \\
\hline Weighted geometric means & $194 \cdot 88$ & 194.96 & $194 \cdot 68$ & \\
\hline $95 \% \mathrm{Cl}$ & $192 \cdot 82,196 \cdot 96$ & $192 \cdot 72,197 \cdot 23$ & $192 \cdot 29,197 \cdot 10$ & \\
\hline Blood $\mathrm{Hg}(\mu \mathrm{g} / \mathrm{l}) \S$ & & & & 0.02 \\
\hline Weighted geometric means & 0.88 & 0.86 & 0.94 & \\
\hline $95 \% \mathrm{Cl}$ & $0.83,0.93$ & $0.81,0.91$ & $0.86,1.01$ & \\
\hline
\end{tabular}

${ }^{*} P$-values were obtained using the $t$ test for the significance of the regression coefficient from the linear regression model with design considered (continuous variable) or the designadjusted Rao-Scott version of Pearson's chi-square test (categorical variable).

† Weighted percentages for considering the stratified multistage probability cluster design.

$\ddagger$ Blood Se data were only available from the NHANES 2011-2016 surveys $(n 7151)$.

$\S 3851$ participants had a missing value of $\mathrm{Hg}(n 11393)$.

Table 2. OR with $95 \% \mathrm{Cl}$ for risk of dyslipidaemia according to tertiles of PUFA intake, a cross-sectional study using data from the NHANES $2009-2016^{*}$ (Odds ratios and $95 \%$ confidence intervals).

\begin{tabular}{|c|c|c|c|c|c|c|c|}
\hline & & & & & & & \\
\hline & & & & & & & \\
\hline & OR & $95 \% \mathrm{Cl}$ & OR & $95 \% \mathrm{Cl}$ & OR & $95 \% \mathrm{Cl}$ & $P$ for trend $T$ \\
\hline PUFA intake $(\mathrm{g} / \mathrm{d})$ & & & & 9.523 & & 33.103 & \\
\hline No. of casesł & & & & & & & \\
\hline Model $1 \S$ & $1.0(\mathrm{Re}$ & & 0.80 & $0.72,0.89$ & 0.79 & $0.70,0.90$ & 0.001 \\
\hline Model 2 & $1.0(\mathrm{Re}$ & & 0.83 & $0.74,0.92$ & 0.80 & $0.70,0.91$ & 0.002 \\
\hline Model 3 & $1.0(\mathrm{Re}$ & & 0.83 & $0.74,0.93$ & 0.80 & $0.71,0.91$ & 0.002 \\
\hline Model 4 & $1.0(\mathrm{Re}$ & & 0.83 & $0.75,0.93$ & 0.81 & $0.71,0.94$ & 0.008 \\
\hline Model $5 a$ & $1.0(\mathrm{Re}$ & & 0.83 & $0.75,0.93$ & 0.82 & $0.71,0.94$ & 0.008 \\
\hline Model $5 b$ & $1.0(\mathrm{Re}$ & & 0.83 & $0.75,0.93$ & 0.81 & $0.71,0.94$ & 0.008 \\
\hline Model $5 c$ & $1.0(\mathrm{Re}$ & & 0.83 & $0.75,0.93$ & 0.81 & $0.71,0.94$ & 0.008 \\
\hline
\end{tabular}

* Design-based multivariable logistic regression models were used to examine the associations of interest.

† $P$ for linear trend was calculated with tertiles coded as 1,2 and 3 as a continuous variable.

‡ Dyslipidaemia was defined as participants who had taken anti-dyslipidaemia medication, or had total cholesterol $\geq 240 \mathrm{mg} / \mathrm{dll}$, or HDL-cholesterol $<40 \mathrm{mg} / \mathrm{dl}$ for males, HDLcholesterol $<50 \mathrm{mg} / \mathrm{dl}$ for females, or LDL-cholesterol $\geq 160 \mathrm{mg} / \mathrm{dl}$, or TAG $\geq 200 \mathrm{mg} / \mathrm{dl}$.

$\S$ Model 1 adjusted for age, sex, and race/ethnicity; model 2 additionally adjusted for education, BMI, smoking status (never, former or current smokers), and alcoholic beverage consumption; model 3 further adjusted for hypertension and diabetes; model 4 further adjusted for dietary intakes of protein and cholesterol; model 5a added blood Se based on model 4; model $5 \mathrm{~b}$ added blood $\mathrm{Hg}$ based on model 4; model $5 \mathrm{c}$ added blood $\mathrm{Se}$ and $\mathrm{Hg}$ based on model 4.

(a)

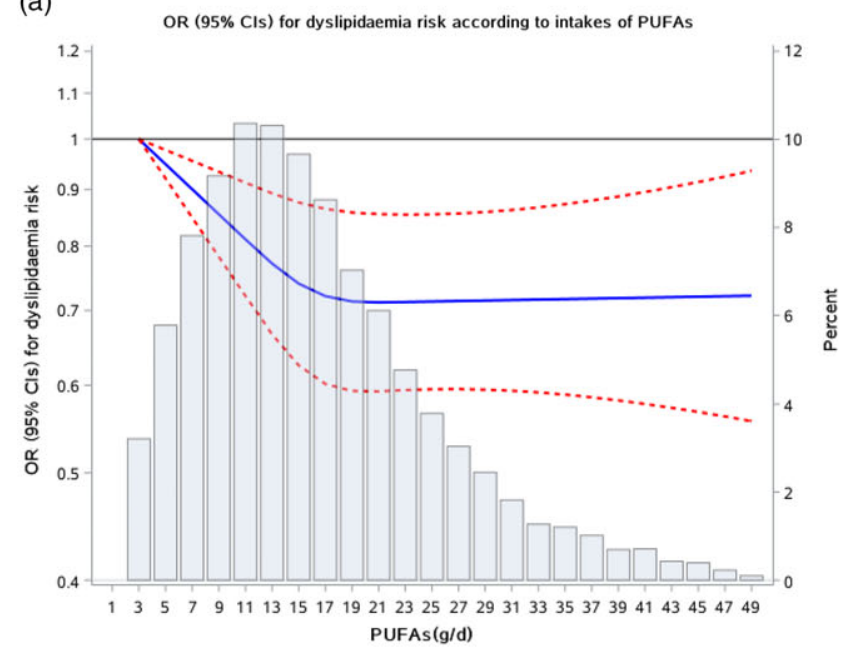

(b)

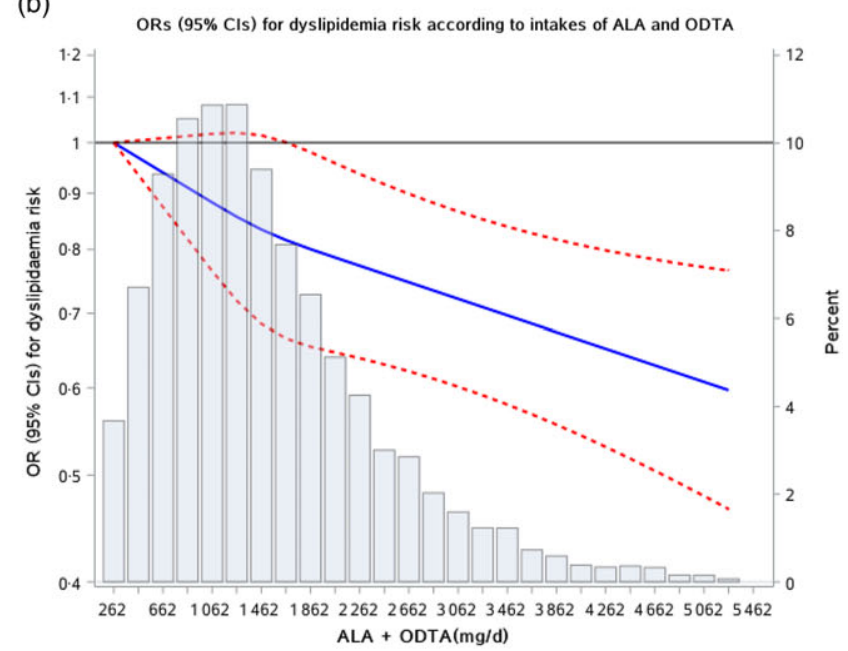

Fig. 2. OR ( $95 \% \mathrm{Cl}$ ) for dyslipidaemia risk according to intakes of PUFA (a) and the sum of ALA and ODTA (b). The association was examined using logistic regression for survey data with the exposure of interest fitted with restricted cubic spline functions. The solid blue lines are OR, and the dashed red lines are $95 \% \mathrm{Cl}$. The light blue bars are histograms of PUFA and the sum of ALA and ODTA, respectively, with the right axis for percentages. ALA, $\alpha$-linolenic acid; ODTA, octadecatetraenoic acid. 
Table 3. OR with $95 \% \mathrm{Cl}$ for risk of dyslipidaemia according to tertiles of $\alpha$-linolenic acid (ALA) plus octadecatetraenoic acid (ODTA) intake, a cross-sectional study using data from the NHANES 2009-2016* (Odds ratios and $95 \%$ confidence intervals).

\begin{tabular}{|c|c|c|c|c|c|c|c|}
\hline & & & & & & & \\
\hline & & & & & & 81) & \\
\hline & OR & $95 \% \mathrm{Cl}$ & OR & $95 \% \mathrm{Cl}$ & OR & $95 \% \mathrm{Cl}$ & $\mathrm{P}$ for trend $\dagger$ \\
\hline ALA and ODTA intake $(\mathrm{mg} / \mathrm{d})$ & & & & .806 & & 6.065 & \\
\hline No. of cases $\ddagger$ & & & & & & & \\
\hline Model $1 \S$ & $1.0(\operatorname{Re}$ & & 0.88 & $0.79,0.99$ & 0.82 & $0.73,0.93$ & 0.002 \\
\hline Model 2 & $1.0(\mathrm{Re}$ & & 0.91 & $0.81,1.02$ & 0.84 & $0.75,0.94$ & 0.004 \\
\hline Model 3 & $1.0(\mathrm{Re}$ & & 0.92 & $0.82,1.03$ & 0.85 & $0.76,0.95$ & 0.006 \\
\hline Model 4 & $1.0(\operatorname{Re}$ & & 0.93 & $0.82,1.05$ & 0.87 & $0.76,0.99$ & 0.04 \\
\hline Model 5a & $1.0(\mathrm{Re}$ & & 0.93 & $0.82,1.05$ & 0.87 & $0.76,0.99$ & 0.04 \\
\hline Model $5 b$ & $1.0(\operatorname{Re}$ & & 0.93 & $0.82,1.05$ & 0.87 & $0.76,0.99$ & 0.04 \\
\hline Model $5 c$ & $1.0(\operatorname{Re}$ & & 0.93 & $0.82,1.05$ & 0.87 & $0.76,0.99$ & 0.04 \\
\hline
\end{tabular}

* Design-based multivariable logistic regression models were used to examine the association of interest.

$\dagger P$ for linear trend was calculated with tertiles coded as 1,2 and 3 as a continuous variable.

$\ddagger$ Dyslipidaemia was defined as participants who had taken anti-dyslipidaemia medication, or had total cholesterol $\geq 240 \mathrm{mg} / \mathrm{dl}$, or HDL-cholesterol $<40 \mathrm{mg} / \mathrm{dl}$ for males, HDLcholesterol $<50 \mathrm{mg} / \mathrm{dl}$ for females, or LDL-cholesterol $\geq 160 \mathrm{mg} / \mathrm{dl}$, or TAG $\geq 200 \mathrm{mg} / \mathrm{dl}$.

$\S$ Model 1 adjusted for age, sex, and race/ethnicity; model 2 additionally adjusted for education, BMI, smoking status (never, former or current smokers), and alcoholic beverage consumption; model 3 further adjusted for hypertension and diabetes; model 4 further adjusted for dietary intakes of protein and cholesterol; model $5 a$ added blood Se based on model 4; model $5 \mathrm{~b}$ added blood $\mathrm{Hg}$ based on model 4; model $5 \mathrm{c}$ added blood Se and $\mathrm{Hg}$ based on model 4.

possible stronger associations were observed. When participants had lower blood se levels, there was a significantly decreased trend of OR for dyslipidaemia along with the increased PUFA intake. The toxicity effect of $\mathrm{Hg}$ included increased oxidative stress, inflammation, and dyslipidaemia ${ }^{(23)}$. Participants with a higher level of blood $\mathrm{Hg}$ had an increased risk of dyslipidaemia. $N-3$ fatty acid intake could antagonise Hg's toxicity ${ }^{(23,37)}$. However, the interrelationship among PUFA intake, blood se and $\mathrm{Hg}$ has not been well elucidated so far.

In Fig. 2(a), the spline's slope changed substantially before and after the turning point of PUFA intake (i.e. about $19 \mathrm{~g} / \mathrm{d}$ ). This slope alteration appeared to be related to differences in the size of relation estimates or different dose-response relationship rather than a lack of association ${ }^{(1,17,38)}$. After the turning point, there was a threshold phenomenon appeared. The main source of PUFA is seafood, especially deep-sea oily fish. As the intake of fish increases, the PUFA are elevated with an initially protective relation but gradually offset with accumulated organic pollutants ${ }^{(39,40)}$. However, the threshold was not observed in the spline result of the sum of ALA and ODTA with dyslipidaemia risk. One possible explanation was that the ALA source was vegetable oil in the American population, especially soyabean oil and rapeseed ${ }^{(41,42)}$, which are less likely to be contaminated by organic pollutants than seafood. Another possible reason for the steady decline for ALA and ODTA was the insufficient dose with a maximum $5.5 \mathrm{~g} / \mathrm{d}$ compared with daily PUFA intake. The continuous declination could also be observed in the spline result of daily PUFA intake in Fig. 2(a) with a dose from minimum to $5.5 \mathrm{~g} / \mathrm{d}$. On the other hand, plant $n-3$ PUFA could be converted to DHA/EPA for functional attributes in metabolism, but this limited conversion resulted in lower biological potency $^{(11)}$.

This research also had some limitations. First, residual confounding from the unmeasured covariates may affect the results from observational data. In the NHANES dataset, there was no information about familial predisposition to dyslipidaemia, which may have potential effect on the risk of dyslipidaemia.
Additionally, data reporting in this research are subject to recall bias and potential selection bias. Moreover, the insignificant results for the sum of DHA, EPA, and DPA may be due to the extremely low consumption level (i.e. $51 \mathrm{mg} / \mathrm{d}$ ) comparing with the recommended daily allowance by the American Heart Association (500 mg for DHA and EPA) ${ }^{(43)}$. In nearly almost dietary PUFA researches ${ }^{(42,44)}, n-6$ PUFA has much higher intake than that of $n$-3 PUFA and ours was no exception. Here, we just focused on total and $n$-3 PUFA intake with the risk of dyslipidaemia. Future interventional studies focusing on each component of PUFA in the daily diet are needed.

From the NHANES 2009-2016 survey data in the US adults, the inverse association between dietary PUFA and dyslipidaemia was observed after adjusting potential confounders, including $\mathrm{Hg}$ and se. Future prospective large-scale longitudinal studies with long-term follow-up are warranted to confirm our findings.

\section{Acknowledgements}

This research received no specific grant from any funding agency, commercial or not-for-profit sectors.

Conceptualisation: J. B., P. X., and J. Z.; data curation: J. Z. and P. X.; formal analysis: J. Z., L. C., S. N., Z. Z., and M. Y.; methodology, J. Z., J. B., and H. Y.; software: J. Z. and L. C.; validation: L. C. and Z. Z.; supervision: J. B. and P. X.; writing - original draft: J. Z., L. C., and S. N.; writing - review and editing: J. B., P. X., Y. Z., and $\mathrm{H}$. Y.

There are no conflicts of interest.

\section{References}

1. Kim SA, Lee JK, Kang D, et al. (2019) Oily fish consumption and the risk of dyslipidemia in Korean adults: a prospective cohort study based on the health examinees gem (HEXA-G) study. Nutrients 11, 2506.

2. Kopin L \& Lowenstein C (2017) Dyslipidemia. Ann Intern Med 167, ITC81-ITC96. 
3. Stocker R \& Keaney JF (2004) Role of oxidative modifications in atherosclerosis. Physiol Rev 84, 1381-1478.

4. Halcox JP, Banegas JR, Roy C, et al. (2017) Prevalence and treatment of atherogenic dyslipidemia in the primary prevention of cardiovascular disease in Europe: EURIKA, a cross-sectional observational study. BMC Cardiovasc Disord 17, 160.

5. Pan L, Yang Z, Wu Y, et al. (2016) The prevalence, awareness, treatment and control of dyslipidemia among adults in China Atherosclerosis 248, 2-9.

6. Writing Group M, Mozaffarian D, Benjamin EJ, et al. (2016) Heart disease and stroke statistics-2016 update: a report from the American Heart Association. Circulation 133, e38-e360.

7. Ference BA, Ginsberg HN, Graham I, et al. (2017) Low-density lipoproteins cause atherosclerotic cardiovascular disease. 1 Evidence from genetic, epidemiologic, and clinical studies. A consensus statement from the European Atherosclerosis Society Consensus Panel. Eur Heart J 38, 2459-2472.

8. Kim MK, Han K, Joung HN, et al. (2019) Cholesterol levels and development of cardiovascular disease in Koreans with type 2 diabetes mellitus and without pre-existing cardiovascular disease. Cardiovasc Diabetol 18, 139.

9. Wikipedia (2019) Polyunsaturated Fatty Acid. https://en wikipedia.org/wiki/Polyunsaturated_fatty_acid_ (accessed November 2020).

10. Lin CR, Chu TM, Luo A, et al. (2019) n-3 polyunsaturated fatty acids suppress metastatic features of human cholangiocarcinoma cells by suppressing twist. $J$ Nutr Biochem 74, 108245.

11. Calder PC (2012) Mechanisms of action of (n-3) fatty acids. J Nutr 142, 592S-599S

12. Silva Figueiredo P, Carla Inada A, Marcelino G, et al. (2017) Fatty acids consumption: the role metabolic aspects involved in obesity and its associated disorders. Nutrients 9, 1158.

13. Berquin IM, Edwards IJ \& Chen YQ (2008) Multi-targeted therapy of cancer by $n-3$ fatty acids. Cancer Lett 269, 363-377.

14. Thota RN, Ferguson JJA, Abbott KA, et al. (2018) Science behind the cardio-metabolic benefits of $n-3$ polyunsaturated fatty acids: biochemical effects $v$. clinical outcomes. Food Funct 9, 3576-3596.

15. Rimm EB, Appel LJ, Chiuve SE, et al. (2018) Seafood long-chain n-3 polyunsaturated fatty acids and cardiovascular disease: a science advisory from the American Heart Association. Circulation 138, e35-47.

16. Sawada T, Tsubata H, Hashimoto N, et al. (2016) Effects of 6-month eicosapentaenoic acid treatment on postprandial hyperglycemia, hyperlipidemia, insulin secretion ability, and concomitant endothelial dysfunction among newly-diagnosed impaired glucose metabolism patients with coronary artery disease. An open label, single blinded, prospective randomized controlled trial. Cardiovasc Diabetol 15, 121

17. Sekikawa A, Cui C, Sugiyama D, et al. (2019) Effect of high-dose marine $n-3$ fatty acids on atherosclerosis: a systematic review and meta-analysis of randomized clinical trials. Nutrients 11, 2599.

18. Berr C, Akbaraly T, Arnaud J, et al. (2009) Increased selenium intake in elderly high fish consumers may account for health benefits previously ascribed to $n-3$ fatty acids. $J$ Nutr Health Aging 13, 14-18.

19. Park K \& Seo E (2017) Toenail mercury and dyslipidemia: interaction with selenium. J Trace Elem Med Biol 39, 43-49.

20. Bulka CM, Persky VW, Daviglus ML, et al. (2019) Multiple metal exposures and metabolic syndrome: a cross-sectional analysis of the National Health and Nutrition Examination Survey 2011-2014. Environ Res 168, 397-405.

21. Ju W, Ji M, Li X, et al. (2018) Relationship between higher serum selenium level and adverse blood lipid profile. Clin Nutr 37, 1512-1517.
22. Houston MC (2007) The role of mercury and cadmium heavy metals in vascular disease, hypertension, coronary heart disease, and myocardial infarction. Altern Ther Health Med $\mathbf{1 3}$, s128-s133.

23. Houston MC (2011) Role of mercury toxicity in hypertension, cardiovascular disease, and stroke. J Clin Hypertens $\mathbf{1 3}$, 621-627.

24. CDC (2019) Centers for Disease Control and Prevention. https://wwwn.cdc.gov/nchs/nhanes/search/datapage.aspx? Component $=$ Laboratory \&CycleBeginYear $=2009$ (accessed November 2019).

25. NHANES (2020) Analytic Notes of Laboratory Test. -https://wwwn.cdc.gov/Nchs/Nhanes/2009-2010/PBCD_F. htm (accessed February 2020).

26. Park SK, Lee S, Basu N, et al. (2013) Associations of blood and urinary mercury with hypertension in US adults: the NHANES 2003-2006. Environ Res 123, 25-32.

27. Backes J, Anzalone D, Hilleman D, et al. (2016) The clinical relevance of $n-3$ fatty acids in the management of hypertriglyceridemia. Lipids Health Dis 15, 118

28. Preston Mason R (2019) New insights into mechanisms of action for $n-3$ fatty acids in atherothrombotic cardiovascular disease. Curr Atheroscler Rep 21, 2.

29. Ganda OP, Bhatt DL, Mason RP, et al. (2018) Unmet need for adjunctive dyslipidemia therapy in hypertriglyceridemia management. J Am Coll Cardiol 72, 330-343.

30. Bays HE, Tighe AP, Sadovsky R, et al. (2008) Prescription $n-3$ fatty acids and their lipid effects: physiologic mechanisms of action and clinical implications. Expert Rev Cardiovasc Ther 6, 391-409.

31. Watanabe Y \& Tatsuno I (2020) Prevention of cardiovascular events with $n$-3 polyunsaturated fatty acids and the mechanism involved. J Atheroscler Thromb 27, 183-198.

32. McKenney JM \& Sica D (2007) Prescription $n$-3 fatty acids for the treatment of hypertriglyceridemia. Am J Health Syst Pharm 64, 595-605.

33. Manson JE, Cook NR, Lee IM, et al. (2019) Marine $n$-3 fatty acids and prevention of cardiovascular disease and cancer. $N$ Engl J Med 380, 23-32.

34. Group ASC, Bowman L, Mafham M, et al. (2018) Effects of $n-3$ fatty acid supplements in diabetes mellitus. NEngl J Med $\mathbf{3 7 9}$, $1540-1550$.

35. Bhatt DL, Steg PG, Miller M, et al. (2019) Cardiovascular risk reduction with icosapent ethyl for hypertriglyceridemia. $N$ Engl J Med 380, 11-22.

36. Yokoyama M, Origasa H, Matsuzaki M, et al. (2007) Effects of eicosapentaenoic acid on major coronary events in hypercholesterolaemic patients (JELIS): a randomised open-label, blinded endpoint analysis. Lancet 369, 1090-1098.

37. Tajik B, Kurl S, Tuomainen TP, et al. (2018) Associations of the serum long-chain $n$-3 PUFA and hair mercury with resting heart rate, peak heart rate during exercise and heart rate recovery after exercise in middle-aged men. Br J Nutr 119, 66-73.

38. Arca M, Borghi C, Pontremoli R, et al. (2018) Hypertriglyceridemia and $n-3$ fatty acids: their often overlooked role in cardiovascular disease prevention. Nutr Metab Cardiovasc Dis 28, 197-205.

39. Ginsberg GL \& Toal BF (2009) Quantitative approach for incorporating methylmercury risks and $n-3$ fatty acid benefits in developing species-specific fish consumption advice. Environ Health Perspect 117, 267-275.

40. Oh R (2005) Practical applications of fish oil ( $n-3$ fatty acids) in primary care. J Am Board Fam Pract 18, 28-36.

41. Baker EJ, Miles EA, Burdge GC, et al. (2016) Metabolism and functional effects of plant-derived $n-3$ fatty acids in humans. Prog Lipid Res 64, 30-56. 
42. Kris-Etherton PM, Taylor DS, Yu-Poth S, et al. (2000) Polyunsaturated fatty acids in the food chain in the United States. Am J Clin Nutr 71, 179S-188S.

43. Richter CK, Bowen KJ, Mozaffarian D, et al. (2017) Total longchain $n-3$ fatty acid intake and food sources in the United States compared to recommended intakes: NHANES 2003-2008. Lipids 52, 917-927.

44. Blasbalg TL, Hibbeln JR, Ramsden CE, et al. (2011) Changes in consumption of $n-3$ and $n$ - 6 fatty acids in the United States during the 20th century. Am J Clin Nutr 93, 950-962. 